\title{
Planning perinatal psychiatry services for
}

\section{Ireland}

\author{
Veronica O'Keane
}

Ir J Psych Med 2010; 27(1): 3-5

The clinical speciality of Perinatal Psychiatry refers to the management of psychiatric disorders that occur during pregnancy and the first year post-partum. Any planning of service development should be based on the needs of the population. Perinatal Psychiatry services, despite the fact that Ireland has the highest birth rate in the EU (www.worldmundi.com), are poorly developed and have not been planned strategically. International data and service development in this field has been restricted by the universal view that psychological distress during pregnancy is not common. Knowledge about the needs that should drive a psychiatric service for women during the perinatal period can neither rely on a substantial academic literature or on service models developed in other countries. There are two caveats to this: novel studies have been published in recent years containing valuable information about the prevalence of perinatal psychiatric morbidity; and we can also borrow expertise from the lessons learned in the UK where perinatal psychiatric service development leads the world.

The following short review will give a brief outline of psychiatric disorders occurring during the perinatal period and possible service models, that could be adopted easily to complement our existing services, to manage these disorders.

\section{Psychiatric disorder during the perinatal period}

Traditionally, descriptions of perinatal psychiatric disorders were confined to disorders that arose during the postpartum period and were classified as the postpartum blues, postpartum depression and puerperal psychosis. In the last 10 years there has been a growing literature and clinical awareness of the problem of depression during pregnancy. It is now known that $50 \%$ of "postnatal depression" commences during pregnancy. Disorders are now better described in terms of their chronological onset; ie, from pregnancy through to the postpartum.

\section{Preconception}

The management of perinatal psychiatric disorders should commence in general psychiatric clinics with all women who are of reproductive age. Contraception and pregnancy should be discussed at the point of diagnosis and the initiation of care planning. Most pregnancies in women with enduring mental illness are unplanned and women should be made aware of the consequences of, firstly, pregnancy and the postpartum

'Veronica O'Keane, Jonathan Swift Clinic, St James's Hospital, Dublin 8, Ireland. Email: vokeane@stjames.ie

*Correspondence

SUBMITTED: FEBRUARY 5, 2010. ACCEPTED: MARCH 2, 2010. as a risk factor for relapse or exacerbation of mental illness and, secondly, possible harmful effects of either discontinuing or continuing prescribed medicines during pregnancies.

\section{Pregnancy as a risk factor for relapse}

Pregnancy is a time of high risk for relapse in women who have a history of affective disorders. A retrospective study in a group of women with bipolar mood disorder reported rates of relapse of approximately $50 \% .{ }^{1}$ A more recent prospective observational study of 201 pregnant women with a history of recurrent major depressive disorder (MDD) reported that $68 \%$ of those who discontinued antidepressant medication after conception relapsed, compared with $26 \%$ of those who continued taking their medication without interruption. ${ }^{2}$ The high rate of relapse in women who remain on medication $(26 \%)$ suggests that pregnancy in itself increases the risk of relapse in women with a history of affective disorder, irrespective of medication discontinuation.

Pregnancy also seems to carry an increased risk for mood disorder in women without a pre-existing diagnosis of affective disorder, as demonstrated by studies examining prevalence rates for depression in general obstetric populations. Gotlib et $\mathrm{al}^{3}$ examined prevalence of MDD in 360 pregnant women and found that $10 \%$ of the women met diagnostic criteria for depression during pregnancy, whilst only $6.8 \%$ were depressed post-partum. In 2001, Evans et $\mathrm{al}^{4}$ published data from the ongoing cohort study of 14,000 women followed from early pregnancy (Avon Longitudinal Pregnancy Cohort Study: ALSPAC), demonstrating that rates of depressive symptomatology, above a threshold suggestive of clinical depression, were $13.5 \%$ at 32 weeks of pregnancy, as opposed to $9.1 \%$ at eight weeks postpartum. Given that rates of depressive disorder in primary care settings have been reported as being $4.8 \%$ to $9.2 \%,{ }^{5}$ it seems that rates of depression in pregnancy are higher than rates in the non-pregnant female population, whilst rates of postpartum depression approximate the general average in women of reproductive age. ${ }^{6}$ In relation to the former, significant specific predictors for antenatal depression are an unwanted or unplanned pregnancy, low social support, low income and history of childhood sexual abuse (CSA). ${ }^{\text {? }}$

\section{Consequences of untreated antenatal depression}

Antenatal depression is associated with poor weight gain, smoking, alcohol and illicit drug use, and poor attendance for obstetric care. ${ }^{8}$ There is now little doubt that severe and persistent psychological stress during pregnancy has a negative impact on obstetric outcome. ${ }^{9}$ Several large-scale population studies have demonstrated a relationship between antenatal psychological stress and either lower birth weight or higher rates of preterm birth. ${ }^{10,11,12,13}$ 
Other studies have shown longer-lasting adverse effects on infant health, with antenatal depression being associated with fearful infant temperament ${ }^{14}$ and developmental delay at 18 months. ${ }^{15}$ The adverse effects of antenatal depression on baby outcome may even persist into adult life. Poor status at birth is now accepted as not only reflective of a more adversive intrauterine environment but also a predictor of chronic disease in adulthood, including coronary heart disease, stroke, type 2 diabetes, the metabolic syndrome and osteoporosis. ${ }^{17,18}$

There is now general acceptance of a prenatal programming hypothesis, mooted initially by Barker's findings of the adult health in a cohort study of babies born during the difficult World War II years. ${ }^{17,18}$ He suggested that metabolic systems were programmed in utero and that a harsh intrauterine environment leads to the development of abnormal "set point" of these systems that remains fixed for life. There is growing evidence implicating prenatal maternal stress in the aetiology of mental illness. There is epidemiological evidence that prenatal stress, specifically hunger and viral infection, may be a risk factor for the development of schizophrenia. ${ }^{19}$ More recently a fetal origins hypothesis for affective disorders invoking prenatal programming of the cortisol stress axis as a consequence of maternal depression has been proposed. ${ }^{20}$ Findings that poorer status at birth, mainly lower birth weight and/or prematurity, and the development of depression from adolescence through to old age, provide further support for this aetiology. ${ }^{16,21,22,23}$

\section{Postnatal psychiatric disorders}

For the mother, depression usually does not remit after the birth of a baby and is a strong predictor of postnatal depression. ${ }^{24}$ Postnatal depression is a subjectively distressing experience for new mothers and babies and can lead to impaired emotional attachment of mother to her baby and severe disruption of family life. The literature on untreated postpartum depression was ground breaking and led the drive for the establishment of Perinatal Psychiatric services in the UK. ${ }^{25}$ It is now accepted that maternal depression in the early life of a baby is associated with poorer than expected developmental outcome in the babies, probably mediated by poor emotional engagement of mother with baby. ${ }^{26}$ Prospective cohort studies in babies whose mothers suffered from postnatal depression, followed through to adulthood, have established that this disadvantage is carried through to adult life with higher than expected rates of externalising disorders (conduct and personality disorder) and internalising disorders (depression and anxiety), in boys and girls respectively. ${ }^{27,28,29}$

Women who experience postpartum depression seem to fall into two main groups in relation to aetiology: those with a typical episode of MDD in the context of a major stressor, birth, and an individual loading of vulnerability factors; and those who have a specific vulnerability to the trigger of childbirth. ${ }^{30,31}$ Depression specific to the postpartum period may be predicted by a short-lived period of elation in the first three days following the birth of the baby, called "postpartum highs". There is also some evidence that this smaller group of women, who seem otherwise not to be vulnerable to becoming depressed, are more likely to have a preceding history of premenstrual dysphoric disorder.

The most severe illness arising during the postpartum period is postpartum psychosis. It is a severe psychosis that usually commences within the first week following the birth of a baby and occurs in women with a bipolar disorder diathesis. It can be predicted to occur in $50 \%$ of primigravid women with a personal history of bipolar disorder: occurring in $30 \%$ without, and $74 \%$ of those with, a family history of puerperal psychosis. $^{32}$

In relation to women with a diagnosis of schizophrenia, rates of childbearing are reduced in general but pregnancy does not appear to confer specific vulnerability to relapse. ${ }^{33}$ They have an increased risk of having either serious obstetric complications or a baby with major congenital malformations. ${ }^{34}$ The majority of women with severe mental illness who have their babies removed, partly or completely, to state care have a diagnosis of schizophrenia.

\section{Perinatal psychiatric services for pregnant women}

Perinatal psychiatric services need therefore to be directed towards providing specialist care for women with pre-existing histories of serious mental illness, specifically towards managing predictable relapses in women with affective disorders during pregnancy and in the prevention of postpartum psychosis. Specialist perinatal services should be available for these women to give preconception advice, advice about pharmacological management of perinatal psychiatric disorder and to liaise with the obstetric and the general psychiatric services about a co-ordinated pregnancy and birth plan.

The second main focus for perinatal psychiatric services is in the detection and management of mood and anxiety disorders that arise in women who have no psychiatric history. Given the high prevalence rates and adverse and persistent outcomes of antenatal depression for mother and baby, screening for depression during pregnancy should be considered. The purpose of any screening programme is to identify undetected illness, or risk of illness, in order to reduce the risks associated with the disease and/or its complications. In the UK, all NHS maternity trusts are advised to implement antenatal screening in order to detect current, past and/or family history of mental health problems. In 2007, the National Institute for Health and Clinical Excellence (NICE UK, 2007) published a clinical guideline on management of antenatal and postnatal mental health. The key priorities include advocating screening questions for 'prediction and detection' at the first contact that pregnant women have with obstetric services, and suggest specific questions to identify depression. The questions are: whether the woman is currently, or has ever been, treated for a mental health problem; has had past or current treatment for depression, or currently feels low in mood. It is also suggested that the use of self-rated screening questionnaires for depression be considered. It could be argued that risk factors specific to perinatal depression be included in this screening process; eg, a history of CSA or PMDD.

This model of screening would be feasible in Ireland as all women are referred to the obstetric services for fetal scanning during pregnancy. Women detected as being at-risk should be discussed at joint psychiatric-obstetric multidisciplinary teams and triaged according to the information available. Decisions about the seriousness of the problem and the need to refer to psychiatric services, specialist or general adult, primary care or social services can then be made. This 
service model requires that specialist perinatal psychiatry services are available that work in an integrated way with the obstetric services. Nurse counselling service, either from the obstetric or psychiatric service, should be an integral part of this team, ensuring that women with significant psychological stress have rapid access to talking therapy. Interpersonal psychotherapy (IPT) for such women has been found to reduce the incidence of postpartum depression. ${ }^{35}$

Studies conducted in Dublin indicate that the rates of postpartum depression are similar to international averages. These findings indicate that affective disorders, for all grades from mild through to severe and enduring, are more likely to occur during pregnancy than at any point in a woman's life. In relation to development of perinatal obstetric services it should be borne in mind that depression is a stress disorder that is related to social disadvantage and poverty. This relationship with social adversity is magnified during pregnancy both among social classes in the economically developed countries and among different regions globally. ${ }^{36}$ Further rationale for input of services to the more socially disadvantaged areas is the relatively higher birth rate in this group.

In summary, psychiatry services should have access to specialist perinatal psychiatry services to help with the management of women with serious mental illness who become pregnant; and obstetric services should have some rudimentary tools for screening for mood disorder during pregnancy. If mood disorder is detected during pregnancy, women should be actively managed, either by primary care services, CMHTs or specialised perinatal psychiatric services. Risk factors for development of depression during pregnancy are similar to those outside of pregnancy and consist largely of an interaction between temperamental vulnerabilities, genetic predisposition and socio-economic stressors. The consequences of untreated mood disorder during pregnancy are poor compliance with obstetric care, increased risk behaviours and possibly poor pregnancy outcome. The evidence that outcome at birth is a major determinant of adult medical and mental health is becoming more compelling and psychiatry can contribute to overall population health for the future in Ireland by developing rational, needs-driven perinatal psychiatric services. Psychiatry should lead the way in convincing health service managers of the need to address the psychological, as well as the medical, needs of pregnant women. Perhaps the most compelling argument for this need is that suicide is now the leading cause of maternal death in the UK. ${ }^{37}$

\section{Declaration of Interest: None.}

\section{References}

1. Viguera $A C$, Nonacs $R$, Cohen LS, Tondo L, Aoife Murray AB, Baldessarini RJ. Risk of Recurrence of Bipolar Disorder in Pregnant and Nonpregnant Women After Discontinuing Lithium Maintenance. American Journal of Psychiatry 2000; 157:179184.

2. Cohen LS, Altshuler LL, Stowe ZN, Faraone SV. Reintroduction of antidepressant therapy across pregnancy in women who previously discontinued treatment. A preliminary retrospective study. Psychother Psychosom 2004; 73:255-258.

3. Gotlib IH, Whiffen VE, Mount JH, Milne K, Cordy NI. Prevalence rates and demographic characteristics associated with depression in pregnancy and the postpartum. Journal of Consulting and Clinical Psychology 1989; 57:269-274.

4. Evans J, Heron J, Francomb H Oke S, Golding J Cohort study of depressed mood during pregnancy and after childbirth. British Medical Journal 2001; 323:257-260.

5. Eberhard-Gran M, Eskild A, Tambs K, Opjordsmoen S, Samuelsen SO. Review of validation studies of the Edinburgh Postnatal Depression Scale. Acta Psychiatr Scand $2001 ; 104 ; 243-249$

6. O'Keane V, Marsh MS. Depression during pregnancy. BMJ 2007; 334:1003-1005. 18. Leigh B, Milgrom J. "Risk factors for antenatal depression, postnatal depression and parenting stress." BMC Psychiatry 8 2008; 24.

7. Leigh B, Milogram J. Risk Factors for antenatal depression, postnatal depression and parenting stress. BMC Psychiatry 8 2008;24

8. Zuckerman B, Amaro H, Bauchner $H$, Cabral $H$. Depressive symptoms during pregnancy: Relationship to poor health behaviors, Am J Obstet Gynecol 1989; 160:1107-1111.

9. Bonari $L$, Pinto N, Ahn E et al. Perinatal risks of untreated depression during pregnancy. Can J Psychiatry 2004; 49:726-735.

10. Copper RL, Goldenberg RL, Das A et al, The preterm prediction study: maternal stress is associated with spontaneous preterm birth at less than thirty five weeks gestation. National Institute of child Health and Human Development Maternal-Fetal Medicine Units Network. Am J Obstet Gynaecol 1996; 175:1286-1292.

11. Hansen $D$, Lou HC, Olsen J. Serious life events and congenital malformations: a national study with complete follow-up. Lancet 2000; 356:875-880.

12. Hedegaard M, Henriksen TB, Secher NJ et al. Do stressful life events affect duration of gestation and risk of preterm delivery? Epidemiology 1996; 7:339-345.

13. Steer RA, Scholl RO, Hediger ML et al. Self-reported depression and negative pregnancy outcomes. J Clin Epidemiol 1992; 45:1093- t099.

14. Zuckerman $B, B$ auchner $H$, Parker $S$, Cabral $H$. Maternal depressive symptoms during pregnancy and newborn irritability. J Dev Behav Pediatr 1990; 11:190-194.

15. O'Connor TG, Heron J, Glover V. Antenatal anxiety predicts child behavioural/ emotional problems independently of postnatal depression. J Am Acad Child Adolesc Psychiatry 2002; 41:1470-1477.

16. O'Connor TG, Ben-Shlomo Y, Heron J, et al. Prenatal anxiety predicts individual differences in cortisol in pre-adolescent children. Biol Psychiatry 2005; 58:211-17.

17. Barker DJP, Winter PD, Osmond $C$ et al. Weight in infancy and death from ischaemic heart disease. Lancet 1989; 2:577-580.

18. Barker DJP, Osmond C, Simmonds SJ, Wield GA. The relation of small head circumference and thinness at birth to death from cardiovascular disease in adult life. British Medical Journal 1993; 306:422-426.

19. Murray RM, Sham P, Van Os J, Zanelli J, Cannon M, McDonald C. A developmental model for similarities and dissimilarities between schizophrenia and bipolar disorder. Schizophr Res 2004; 71:405-416.

20. O'Keane V, Scott J. From 'obstetric complications' to a maternal-foetal origin hypothesis of mood disorder. British Journal of Psychiatry 2005; 186:367-368.

21. Barker DJP, Osmond C, Rodin I, Fall CHD, Winter PD. Low weight gain in infancy and suicide in adult life. BMJ 1995; 311:1203.

22. Patton GC, Coffey C, Carlin JB, Olsson CA, Morley R. Prematurity at birth and adolescent depressive disorder. The British Journal of Psychiatry 2004; 184:446-447. 23. Thompson C, Syddall H, Rodin I, Osmond C, Barker DJP. Birth weight and the risk of depressive disorder in late life. British Journal of Psychiatry 2001; 179:450-455. 24. Evans J, Heron J, Francomb H, Oke S, Golding J, Cohort study of depressed mood during pregnancy and after childbirth. British Medical Journal 2001; 323:257-260.

25. Murray L, Hipwell A, Hooper R et al, The cognitive development of 5-year-old children of postnatally depressed mothers. J Child Psychol Psychiatry 1996; 37:927935.

26. Murray L, Cooper PJ, Wilson A, Romaniuk H. Controlled trial of the short-and long-term effect of psychological treatment of postpartum depression: 2. Impact on the mother-child relationship and child outcome. British Journal of Psychiatry 2003; 182:420-427.

27. Pawlby, S, et al. "Antenatal depression predicts depression in adolescent offspring Prospective longitudinal community-based study." J.Affect.Disord 2008. 28. Hay DF, Pawlby S, Sharp D et al. Intellectual problems shown by 11 -year-old children whose mothers had postnatal depression. J Child Psychol Psychiat 2001; 42:871-889.

29. Hay DF, Pawlby S, Angold A, Harold GT, Sharp D. Pathways to violence in children of mothers who were depressed postpartum. Dev Psychol 2003; 39:1083-1094.

30 . Forty L, Jones L, McGregor S et al. Familiarity of postpartum depression in unipolar disorder: Results of a family study. American Journal of Psychiatry 2006; 163: 1549 53

31. O'Keane V. Mood disorder during pregnancy: aetiology and management Psychiatric Disorders in Pregnancy. P 69-107. Eds, O'Keane V, Marsh M, Seneviratne G. Taylor \& Francis: 2006.

32. Jones I, Craddock N, et al. Familiality of the Puerperal Trigger in Bipolar Disorder: Results of a Family Study. Am J Psychiatry 2001; 158:913-917

33. Howard LM, Goss C, Leese M, Thornicroft G, Medical outcome of pregnancy in women with psychotic disorders and their infants in the first year after birth. British Journal of Psychiatry 2003; 182:63-67.

34. Nilsson $E$, Lichtenstein $\mathrm{P}$, Cnattingius $\mathrm{S}$, Murray RM, Hultman CM Women with schizophrenia: pregnancy outcome and infant death among their offspring. 2002; 58:221-229

35. Spinelli MG, Endicott J. Controlled clinical trial of interpersonal psychotherapy versus parenting education program for depressed pregnant women. Am J Psychiatry $2003 ; 160: 555-562$.

36. Patel $V$ et al. Effect of maternal mental health on infant growth in low income countries: new evidence from South Asia, BMJ 2004; 328:820-823.

37. Oates M. Suicide: the leading cause of maternal death. British Journal of Psychiatry 2003; 183:279-281. 